\title{
Modelagem magnética de fontes elipsoidais
}

\author{
Diego T. Tomazella*(ON/MCTI), Vanderlei C. Oliveira Jr. (ON/MCTI)
}

Copyright 2016, SBGf - Sociedade Brasileira de Geofísica

Este texto foi preparado para a apresentação no VII Simpósio Brasileiro de Geofísica, Ouro Preto, 25 a 27 de outubro de 2016. Seu conteúdo foi revisado pelo Comitê Técnico do VII SimBGf, mas não necessariamente representa a opinião da SBGf ou de seus associados. É proibida a reprodução total ou parcial deste material para propósitos comerciais sem prévia autorização da SBGf.

\section{Resumo}

In this work we will present results from a numerical modeling of the magnetic field and total field anomaly, represented by triaxial ellipsoid sources. Such approach provide analytical results for anisotropy of magnetic susceptibility as well as for self-demagnetization effects, which can be easily adapted for distinctive geologic structures - hence being an useful tool for educational (e.g., potential methods and rock magnetism) and applied geophysics (e.g., characterization of high magnetic susceptibility, mineralized bodies) purposes. Numerical tests by means of a Python code (currently under development) allowed us to compared the effects of different geometries (ellipsoidal sources, spheres, dipole lines and horizontal cylinders), which were used to validate our computational implementation. This code will be freely available to the scientific community by the end of the year.

\section{Introdução}

Na década de 1970, Farrar (1979) mostrou a utilidade do modelo elipsoidal na investigação geofísica de exploração aplicada ao estudo de anomalias magnéticas sobre uma mina de ouro localizada em Tennant Creek, Australia. A forma de um elipsoide se mostrou a mais competente para modelar os corpos em formatos de tubos lenticulares, e também o mais apropriado para lidar com a desmagnetização de corpos de alta susceptibilidade - corpos com susceptibilidade magnética acima de $0.1 \mathrm{SI}$ (Clark, 2014).

Em seu livro A Treatise on Electricity and Magnetism, Maxwell (1881) mostrou que apenas formas delimitadas por superfícies de segundo grau são magnetizadas uniformemente quando colocadas sobre um campo uniforme. No caso do elipsoide, como o campo interno é independente das coordenadas espaciais sua magnetização é completamente homogênea, tornando-a a única forma geométrica que possui solução verdadeiramente analítica para a desmagnetização (Clark et al., 1986).

A modelagem do campo magnético produzido por um elipsoide no domínio do espaço foi apresentada pela primeira vez de modo completo por Emerson et al. (1985) em conjunto com uma compilação de modelagens de diversas formas geométricas. Através de uma notação consistente este trabalho supriu a falta do modelo elipsoidal da literatura. Este modelo, porém, consistia na divisão entre elipsoides prolatos e oblatos com pequenas diferenças no seu algoritmo. Na implementação deste trabalho, utilizaremos um modelo generalizado para elipsoides triaxiais publicado por Clark et al. (1986). A partir da solução do potencial gravimétrico para elipsoides uniformes, formalmente resolvido por Dirichlet (1839) em seu artigo Sur un nouvelle methode pour la determination des integrales multiples que usa as integrais elípticas normais de primeira e segunda ordem de Legendre, juntamente com a relação de Poisson (Grant and West, 1965) é possível calcular o potencial magnético, e posteriormente o campo magnético gerado pelo corpo através do gradiente deste potencial.

Apesar da grande aplicabilidade do modelo elipsoidal na geofísica, apenas alguns softwares proprietários possuem sua implementação. Neste sentido, este trabalho tem a finalidade de disponibilizar uma ferramenta de código aberto, para o cálculo do campo magnético a da anomalia de campo total gerado por elipsoides, que poderá ser utilizada livremente por toda a comunidade geofísica.

\section{Metodologia/ Problema Investigado}

A indução magnética total $B_{i}$ pode ser escrita como:

$$
B_{i}=\sum_{j=1}^{L} b_{i}^{j}, i=1, \ldots, N
$$

Sendo $b_{i}^{j}$ a indução magnética produzida pelo j-ésimo elipsoide, $j=1, \ldots, L$ e que pode ser escrito como:

$$
b_{i}^{j}=2 \pi \times a_{j} b_{j} c_{j} \times\left[M_{i}^{j}-D_{i}^{j}\right] \times J^{j}
$$

Onde os valores de semi-eixos principais $a_{j}, b_{j}$ e $c_{j}$ definem as dimensões dos elipsoides, o vetor de magnetização $J^{j}$ sendo dado por: 


$$
J^{j}=\left[\begin{array}{c}
J_{1 j} \\
J_{2 j} \\
J_{3 j}
\end{array}\right]_{3 \times 1}
$$

$\mathrm{E} M_{i}^{j}$ a matriz:

$$
M_{i}^{j}=\frac{1}{\sqrt{\left[\left(a^{2}+\lambda\right)\left(b^{2}+\lambda\right)\left(c^{2}+\lambda\right)\right]}}\left[\begin{array}{llll}
\frac{\partial \lambda}{\partial x_{1}} \frac{x_{1}}{a^{2}+\lambda} & \frac{\partial \lambda}{\partial x_{1}} \frac{x_{2}}{b^{2}+\lambda} & \frac{\partial \lambda}{\partial x_{1}} \frac{x_{3}}{c^{2}+\lambda} \\
\frac{\partial \lambda}{\partial x_{2}} \frac{x_{1}}{a^{2}+\lambda} & \frac{\partial \lambda}{\partial x_{2}} \frac{x_{2}}{b^{2}+\lambda} & \frac{\partial \lambda}{\partial x_{2}} \frac{x_{3}}{c^{2}+\lambda} \\
\frac{\partial \lambda}{\partial x_{3}} \frac{x_{1}}{a^{2}+\lambda} & \frac{\partial \lambda}{\partial x_{3}} \frac{x_{2}}{b^{2}+\lambda} & \frac{\partial \lambda}{\partial x_{3}} \frac{x_{3}}{c^{2}+\lambda}
\end{array}\right]_{3 \times 3}
$$

Que contém as derivadas da função $\lambda$ em cada um dos eixos do sistema de coordenadas do elipsoide $\left(x_{1}, x_{2}, x_{3}\right)$ que possui sua origem (posição $0,0,0$ ) no centro do corpo. A função $\lambda$ representa a maior raiz real da equação geométrica de um elipsoide com um parâmetro de controle de forma $s$ (coordenadas elipsoidais $\lambda, \mu, v$ que representam as raízes maior, intermediaria e menor respectivamente):

$\frac{x_{1}^{2}}{\left(a^{2}+s\right)}+\frac{x_{2}^{2}}{\left(b^{2}+s\right)}+\frac{x_{3}^{2}}{\left(c^{2}+s\right)}=1 \quad(s=\lambda, \mu, v)$

Para a posição $x_{1}$ temos como derivada em relação a $\lambda$ :

$$
\frac{\partial \lambda}{\partial x_{1}}=\frac{2 /\left(a^{2}+\lambda\right)}{\left(\frac{x_{1}}{a^{2}+\lambda}\right)^{2}+\left(\frac{x_{2}}{b^{2}+\lambda}\right)^{2}+\left(\frac{x_{3}}{c^{2}+\lambda}\right)^{2}}
$$

As outras derivadas espaciais são facilmente obtidas por simetria.

Já matriz $D_{i}^{j}$ é:

$$
D_{i}^{j}=\left[\begin{array}{ccc}
A(\lambda) & 0 & 0 \\
0 & B(\lambda) & 0 \\
0 & 0 & C(\lambda)
\end{array}\right](7)
$$

Definida pelas integrais do potencial de um elipsoide homogêneo:

$$
\begin{aligned}
& A(\lambda)=\int_{\lambda}^{\infty} \frac{d u}{\left(a^{2}+u\right) R(u)}(8) \\
& B(\lambda)=\int_{\lambda}^{\infty} \frac{d u}{\left(b^{2}+u\right) R(u)}(9) \\
& C(\lambda)=\int_{\lambda}^{\infty} \frac{d u}{\left(c^{2}+u\right) R(u)}(10)
\end{aligned}
$$

Onde $R(u)$ é uma função representada por:

$$
R(u)=\sqrt{\left(a^{2}+u\right)\left(b^{2}+u\right)\left(c^{2}+u\right)}
$$

Para o cálculo das integrais foi utilizado soluções analíticas conhecidas como integrais elípticas normais de Legendre de primeira e segunda ordem, para obter uma melhor eficiência computacional.

Para incluir a anisotropia e a desmagnetização o vetor de magnetização resultante deve ser calculado por:

$\overline{J_{r}}=[I+K N]^{-1}\left(K \bar{F}+\bar{J}_{N R M}\right)$

Onde $I$ é uma matriz identidade, $K$ a matriz de susceptibilidades e $\bar{J}_{N R M} \circ$ vetor de magnetização remanente.

Já $N$ é uma matriz $3 \times 3$ que contém os fatores de desmagnetizações que podem ser calculados de forma analítica em elipsoides:

$$
\begin{aligned}
& N_{1}=\frac{4 \pi a b c}{\left(a^{2}-b^{2}\right)\left(a^{2}-c^{2}\right)^{0.5}}[F(k, \theta)-E(k, \theta)] \\
& N_{2}=\frac{4 \pi a b c\left(a^{2}-c^{2}\right)^{0.5}}{\left(a^{2}-b^{2}\right)\left(b^{2}-c^{2}\right)}\left[E(k, \theta)-\left(\frac{\left(b^{2}-c^{2}\right)}{\left(a^{2}-c^{2}\right)}\right) F(k, \theta)-\frac{c\left(a^{2}-b^{2}\right)}{a b\left(a^{2}-c^{2}\right)^{0.5}}\right]
\end{aligned}
$$




$$
N_{3}=\frac{4 \pi a b c}{\left(b^{2}-c^{2}\right)\left(a^{2}-c^{2}\right)^{0.5}}\left[\frac{b\left(a^{2}-c^{2}\right)^{0.5}}{a c}-E(k, \theta)\right](15)
$$

Cada coluna da matriz $N$ corresponde aos valores de $N_{1}, \quad N_{2}$ e $N_{3}$ respectivamente. Já $F(k, \theta)$ e $E(k, \theta)$, são novamente as integrais elípticas normais de Legendre de primeira e segunda ordem.

Importante notar que toda a modelagem direta ocorre no sistema de coordenadas do corpo, sendo necessário ao final passar novamente para as coordenadas geográficas os resultados obtidos.

\section{Resultados}

Os resultados apresentados a seguir apresentam uma comparação entre a anomalia de campo total produzida por fontes elipsoidais e a anomalia produzida por outras formas geométricas com o intuito de fazer uma validação numérica da nossa implementação computacional. É importante salientar que nestas figuras o eixo $x$ está alinhado com o norte verdadeiro, o eixo y aponta para leste e o eixo $z$ aponta para baixo.

$\mathrm{Na}$ figura 1, comparamos a anomalia de campo total produzida por um elipsoide e com aquela produzida por uma esfera. As fontes são descritas pelos seguintes parâmetros:

\begin{tabular}{|c|c|}
\hline Parâmetros & Valores \\
\hline $\mathrm{a}, \mathrm{b}, \mathrm{c}$ & $5.501,5.500,4.999(\mathrm{~m})$ \\
\hline Alfa, delta, gama & $180^{\circ}, 0^{\circ}, 45^{\circ}$ \\
\hline Centro do elipsoide $(\mathrm{x}, \mathrm{y}, \mathrm{z})$ & $0,0,80(\mathrm{~m})$ \\
\hline Magnetização remanente $^{*}$ & $100(\mathrm{nT}), 90^{\circ}, 0^{\circ}$ \\
\hline Campo da Terra $^{*}$ & $60000(\mathrm{nT}), 37^{\circ}, 85^{\circ}$ \\
\hline $\mathrm{K}_{1}{ }^{*}$ & 0.1 (c.g.s), $90^{\circ}, 0^{\circ}$ \\
\hline $\mathrm{K}_{2}{ }^{*}$ & 0.1 (c.g.s), $180,0^{\circ}$ \\
\hline $\mathrm{K}_{3}{ }^{*}$ & 0.1 (c.g.s), $0^{\circ}, 90^{\circ}$ \\
\hline
\end{tabular}

Tabela 1: Parâmetros do elipsoide modelado para a resposta gerada na figura 1 . *Valores referentes à intensidade, inclinação $e$ declinação respectivamente.

Como resposta a esses parâmetros, 0 vetor de magnetização resultante do elipsoide foi de $4045.40 \mathrm{nT}$, $35.67^{\circ}$ e $85.42^{\circ}$ de intensidade, inclinação e declinação respectivamente.

Dessa forma foi modelado, utilizando o software Fatiando a Terra (Uieda et al., 2013), a anomalia de campo aproximada para uma esfera com esse mesmo vetor de magnetização resultante e um raio de 5.5 metros e seus resultados comparados.

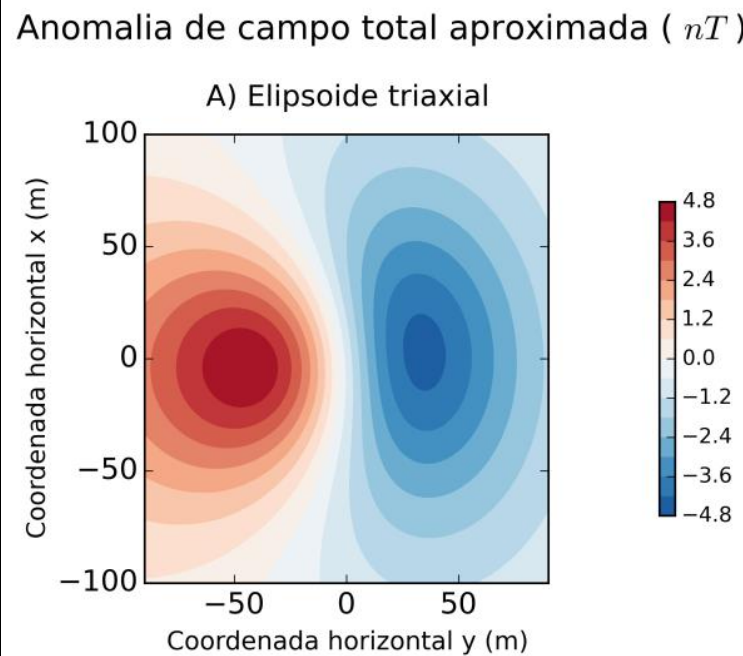

B) Esfera

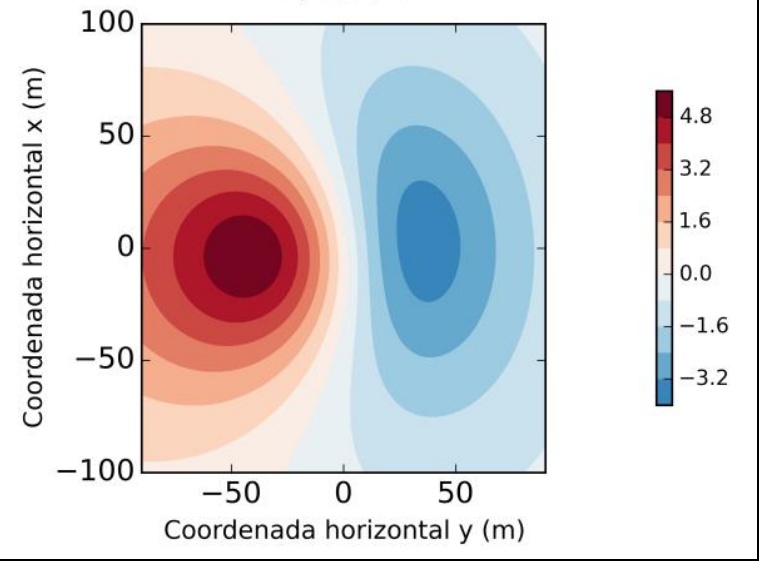

Figura 1: Anomalia de campo aproximada para: A) um elipsoide triaxial e B) uma esfera.

$\mathrm{Na}$ figura 2, modelamos um elipsoide com um semi-eixo maior bastante alongado de forma a simular um cilindro. Como comparação utilizamos a modelagem proposta por Emerson et al. (1985) de uma elipse cilíndrica 2D e uma linha de dipolos formada por esferas de raio 1 metro modeladas também pelo software livre Fatiando a Terra (Uieda et al., 2013). Os parâmetros para essa modelagem foram:

\begin{tabular}{|c|c|}
\hline Parâmetros & Valores \\
\hline a,b,c & $9000,1.01,0.99(\mathrm{~m})$ \\
\hline Alfa, delta, gama & $270^{\circ}, 0^{\circ}, 90^{\circ}$ \\
\hline Centro do elipsoide $(\mathrm{x}, \mathrm{y}, \mathrm{z})$ & $0,0,80(\mathrm{~m})$ \\
\hline Magnetização remanente* $^{*}$ & $1000(\mathrm{nT}), 90^{\circ}, 0^{\circ}$ \\
\hline Campo da Terra* $^{*}$ & $1(\mathrm{nT}), 90^{\circ}, 0^{\circ}$ \\
\hline
\end{tabular}




\begin{tabular}{|l|l|}
\hline $\mathrm{K}_{1}{ }^{*}$ & 0.01 (c.g.s), $90 \circ, 0 \circ$ \\
\hline $\mathrm{K}_{2}{ }^{*}$ & 0.01 (c.g.s), $180,0^{\circ}$ \\
\hline $\mathrm{K}_{3}{ }^{*}$ & 0.01 (c.g.s), 0 ○, $90^{\circ}$ \\
\hline
\end{tabular}

Tabela 2: Parâmetros do elipsoide modelado para a resposta gerada na figura 2. *Valores referentes à intensidade, inclinação $e$ declinação respectivamente.

Assim, o vetor de magnetização resultante do elipsoide foi de $940.34 \mathrm{nT}, 90^{\circ}$ e $0^{\circ}$ de intensidade, inclinação e declinação respectivamente. Utilizando esses valores para o cálculo da indução magnética gerado pela linha de dipolos obtivemos o resultado apresentado na figura 2 .

\section{Discussão e Conclusões}

A modelagem direta do campo magnético gerado por fontes elipsoidais se mostrou satisfatória para os testes conduzidos neste trabalho. Colocando os valores dos semi-eixos próximos uns dos outros, aproximamos o elipsoide para uma esfera e encontramos valores perto da indução magnética causada por uma esfera. Alongando o semi-eixo maior, mostramos que o campo magnético produzido pelo elipsoide se aproxima daquele produzido por um cilindro e por uma linha de dipolos. Os resultados serviram para validar a nossa implementação computacional e para demonstrar a flexibilidade do modelo elipsoidal na representação de diferentes formas geométricas. Em todas as simulações apresentadas, contudo, considerou-se que a susceptibilidade magnética é isotrópica. Testes numéricos mais complexos serão desenvolvidos nas próximas etapas deste projeto de pesquisa.

\section{Agradecimentos}

O autor Diego Takahashi Tomazella agradece ao CNPq pela concessão da bolsa de mestrado.

\section{Referências}

Blakely, R. J. (1996). Potential theory in gravity and magnetic applications. Cambridge University Press.

Clark, D., Saul, S., and Emerson, D. (1986). Magnetic and gravity anomalies of a triaxial ellipsoid. Exploration Geophysics, 17(4): 189-200.

Clark, D. A. (2014). Methods for determining remanent and total magnetisations of magnetic sources-a review. Exploration Geophysics, 45(4): 271-304.
Anomalia de campo total aproximada ( $n T)$

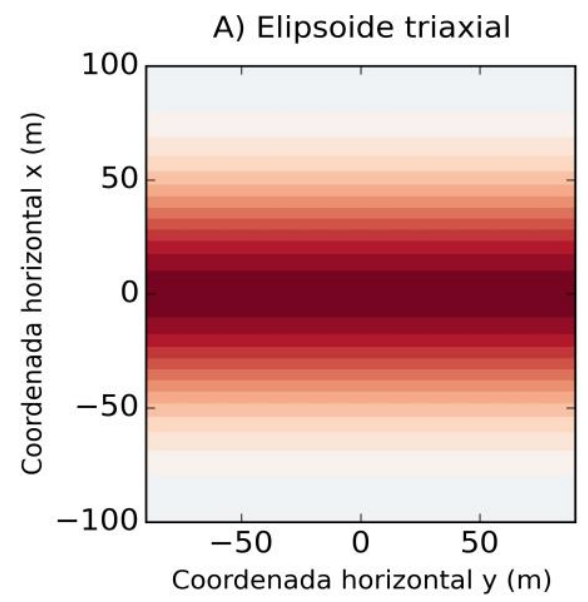

B) Linha de dipolos

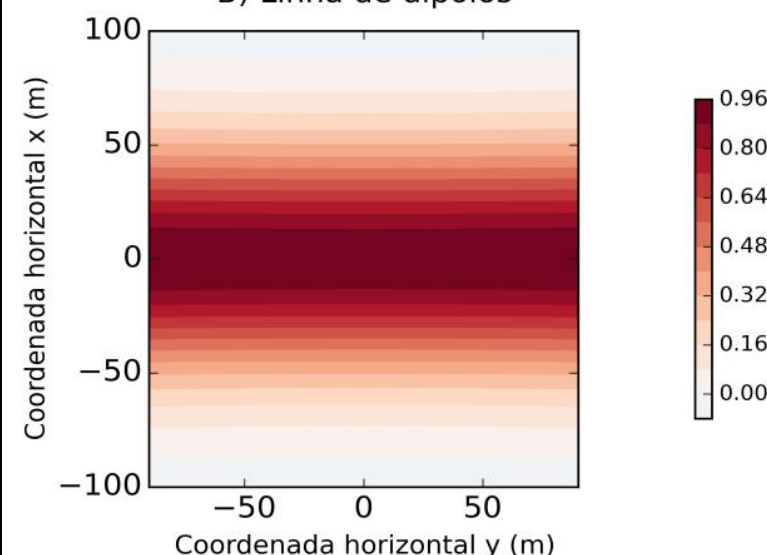

C) Elipse Cilindrica 2D

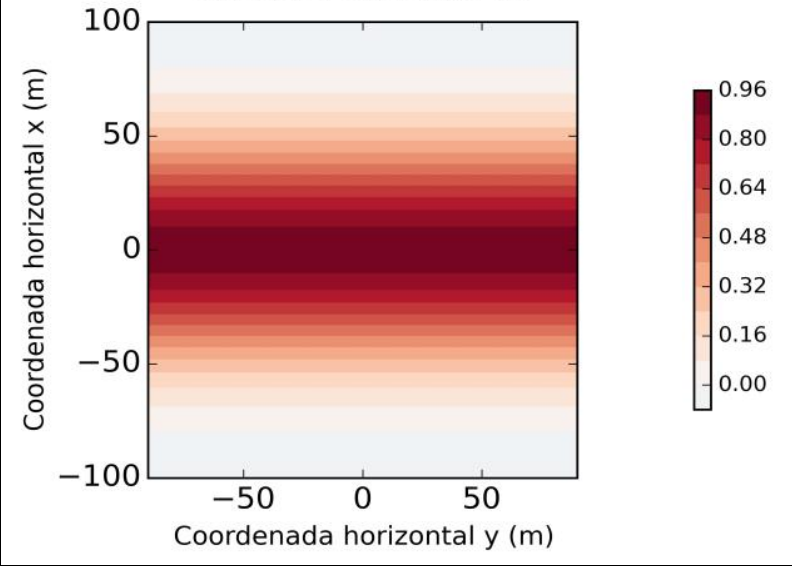

Figura 2: Anomalia de campo aproximada para: A) um elipsoide triaxial alongado na direção do eixo y, B) uma linha de dipolos formada por um conjunto de esferas e C) uma elipse cilíndrica $2 D$. 
Dirichlet, P. G. L. (1839). Sur un nouvelle methode pour la determination des integrales multiples. Comp. Rend. Acad. Science, 8: 156-160.

Emerson, D. W., Clark, D., and Saul, S. (1985). Magnetic exploration models incorporating remanence, demagnetization and anisotropy: $\mathrm{Hp} 41 \mathrm{c}$ handheld computer algorithms. Exploration Geophysics, 16(1): 1122.

Farrar, L. (1979). Some comments on detailed magnetic investigations of ellipsoidal bodies at tennant creek. Exploration Geophysics, 10(1): 26-33.

Grant, F. S. andWest, G. F. (1965). Interpretation theory in applied geophysics. McGraw-Hill Book.

Hanna, W. (1924). Some historical notes on early magnetic surveying in the us geological survey. Geologic applications of modern aeromagnetic surveys: US Geological Survey Bulletin, p. 63-73.

Maxwell, J. C. (1881). A treatise on electricity and magnetism, volume 1 . Clarendon press.

Nabighian, M. N., Grauch, V., Hansen, R., LaFehr, T., Li, Y., Peirce, J., Phillips, J., and Ruder, M. (2005). The historical development of the magnetic method in exploration. Geophysics, 70(6): 33-61.

Oliveira Jr, V., Sales, D., Barbosa, V., and Uieda, L. (2015). Estimation of the total magnetization direction of approximately spherical bodies. Nonlinear Processes in

Geophysics, 22(2): 215-232.

Reford, M. and Sumner, J. (1964). Aeromagnetics. Geophysics, 29(4): 482-516.

Uieda, L., V. C. Oliveira Jr, and V. C. F. Barbosa (2013), Modeling the Earth with Fatiando a Terra, Proceedings of the 12th Python in Science Conference, pp. 91 - 98. 